\title{
The Clinical Infection Trend, Drug Resistance and Nursing Intervention Measures of Acinetobacter Baumannii in Pediatric Intensive Care Unit
}

\author{
Weili Li, Wenwen Liu ${ }^{a}$ * \\ Department of Nursing, Zhengzhou People's Hospital, Zhengzhou, 450053, China \\ azmby519@163.com
}

Keywords: PICU; ABA; Drug resistance; Nursing intervention.

\begin{abstract}
Objective to explore the clinical infection trend, drug resistance and nursing intervention measures of Acinetobacter baumannii (Acinetobacter baumannii, ABA) in the pediatric intensive care unit (pediatric intensive care unit, PICU). Methods the separation rate and drug resistance of ABA in various clinical specimen of sick children diagnosed with ABA infection from February 2012 to December 2014 were retrospectively analyzed. Results 212 cases of sick children were infected with $\mathrm{ABA}$ and 329 strains of ABA were detected during the 3 years. It showed that the drug resistance rate of $\mathrm{ABA}$ against Amikacin, Levofloxacin and Ciprofloxacin was at the lowest $<3$. $0 \%$, while it was at the highest $>70.0 \%$ against Penicillins, Cephalosporins and other antibiotic drugs and the rising trend was keep going. Conclusion the ABA infection in PICU kept increasing. And the common-used antibacterial agents was with serious drug resistance and multi-drug resistance, which was keeping rising. According to the bacterial culture and drug sensitivity findings, it could control the colonization and spreading of ABA in the PICU positively to use drug reasonably and scientifically, to strengthen the management of the hospital environment and personnel sterilization and to take effective nursing intervention measures.
\end{abstract}

\section{Introduction}

Acinetobacter baumannii (ABA) is widely distributed in the natural environment, and it is easy to survive in various humid environments in hospital for a long time. In recent years, with the wide application of broad-spectrum antimicrobial agents, ABA-induced infection increased greatly, multi-drug resistant strains appeared and grew rapidly, especially in the ICU, which was more and had already got attention in clinic[1]. The detection rate of ABA and the number of multi-drug resistant strains increased continuously in children in pediatric intensive care unit (PICU) because of the low immune function, severe disease, long-time hospitalization, various intubation tube and catheter, mechanical ventilation, invasive procedures and other factors, which did a great harm to the children. The ABA clinical features isolated from various clinical specimens in PICU from February 2012 to December 2014 were retrospectively analyzed, it concluded that the disinfection and isolation in the ward and effective nursing measures could positively control the colonizing and spreading of the drug-resistant strains in PICU.

\section{Material and methods}

\subsection{Clinical information}

212 cases diagnosed with the ABA infection from February 2012 to December 2014 were selected. Among them, there were 116 male cases and 96 female cased with the age ranging from one month to five years old ( an average age of 5.72years old \pm 3.12 years old). And there were 120 cases with endotracheal intubation, 191 cases with indwelling gastric tube, 8 cases with indwelling catheter and 45 cases with peripheral central venous catheter (PICC). And the hospital stays was ranging from 11days to 83days with the average of 21.8 days.

\subsection{Methods}

The bacterial culture findings of various clinical specimens in children infected with ABA were retrospectively analyzed, at the same time the relative information of children were collected. The 
microorganism automatic identification system of VITEK-32 produced by France Biological Merry Emmanuel Company was applied to the identification of bacteria and Kindy-Bauer was applied to the drug sensitive test.

\section{Results}

\subsection{The detection rate of $\mathrm{ABA}$}

The total 329 ABA strains were checked out during 3 years, mostly from sputum and bronchial secretions, taking up $89.4 \%$. There were 45 strains in the year of 2007 , accounting for $9.6 \% ; 74$ strains in the year of 2008, accounting for 12.7\%; 210 strains in 2009 years, accounting for 24. 3\%.

\subsection{The drug resistance rate of the common-used antibacterial agents}

329 strains of ABA detected out were resistant against the clinical commonly used antibiotics at different degrees. Among them, the resistance against more than 3 kinds of antibiotics took up the majority. The resistance rates of penicillins and cephalosporins and other antibiotics were all more than $70.0 \%$ respectively. The resistance rates of Amikacin, levofloxacin, ciprofloxacin were at the lowest less than 3.0\%. The resistance rates of imipenem and meropenem rose from $35.7 \%$ and $35.1 \%$ in 2007 to $74.9 \%$ and $78.5 \%$ respectively in the year of 2009 , in addition, the total resistant rates were at a higher level, with $62.8 \%$ and $64.6 \%$ respectively. It indicated a high resistance rate and multi-drug resistance, and the resistance rate was with a rising trend.

\section{Discussion}

\subsection{Risk factors of ABA infection}

Since the children treated in PICU suffered the severe disease, low immunity, long-time hospital stays, heavy use of antibacterial drugs, tracheotomy, endotracheal intubation, use of breathing machine, indwelling gastric tube and many invasive procedures like the imbedding of the PICC tube, their protective barriers were broken down which made them become the high risk group infecting with ABA and the ABA infection increased gradually. The findings showed that the infectious rate and detection rate of ABA in PICU in our hospital increased year on year since 2007. The ABA infection in hospital was mainly the acquired respiratory infection, especially the infection related to the mechanical ventilation, gathered in ICU and Respiratory Department [2]. These factors lead to a large amount of the specimen detection of sputamentum and tracheal aspirates, and a high rate of the bacterial strain detection, which should be paid more attention.

Broad-spectrum antibacterials were widely used in PICU, which increased the colonization and reproduction of the multi-resistant ABA. The dynamic evolution of the clinical common-used antibacterial agent resistance in PICU of our hospital from February 2012 to December 2014 was analyzed in this study. The findings showed that the common-used antibacterial agents resistance in general was on the rise, and the resistance rates of the penicillins, cephalosporins or $\beta$-lactam were all more than $70.0 \%$. And the common-used antibacterial agents resistance in general was on the rise.

\subsection{Nursing intervention measures}

Cleaners training in PICU should be paid attention. The hospital cleaners are all from migrant workers, laid-off workers, housewives, etc., they lack health common sense. Although they have received training by Cleaning Company before they are on their posts. However, the requirement of the PICU is much higher than the the general wards. Therefore, it is important to train the cleaners responsible for the department for the basic information on the disinfection and isolation.

The cleaning and disinfection work should be strengthened. The cleaning and disinfection in the bed units and wards environment in the department should be strengthened. The windows should be opened regularly to have a good ventilation to keep a fresh air in the ward. The work staff should change dresses, shoes and wear hats and masks before entering the PICU. And the children should take the terminal disinfection according to the requirement when they leave hospital or transfer to other departments. 
The isolation system at the bedside should be seriously implemented. The children suspected or diagnosed with the ABA infection, should be equipped with thermometer, spray pipe separately and be the last one to receive treatment. The medical tools and hands should be immediately disinfected after the operation of the diagnosis and nursing. The hands of the medical staff are the main media of the infection in hospital, which should be paid the most attention. And the medical staff should positively implement the hand hygiene, without a chance or psychological lacking in vigilance. The children with low immunity should be taken the protective isolation measures.

Aseptic technique operation should be strictly obeyed. Since the ABA infection is closely related to the mechanical ventilation infection, the disinfection and isolation management of mechanical ventilation in children should be strengthened. There are 120 cases with trachea cannula, accounting for $56.6 \%$ in this study. Therefore, various invasive operation should be strictly operated with the aseptic technique, especially for sputum suction to prevent from infection.

Antimicrobial drugs should be reasonably selected. Since the lower respiratory tract is the main source of ABA, its secretions should be conducted sputum culture in order to know the children infectious information and the treatment effect of the antibacterial agents. The drugs should be used scientifically and reasonably according to the children bacterial culture and the drug sensitivity findings to avoid the increase of the antibiotic drug resistance [3, 4].

\section{Conclusion}

Strengthening the control measures against hospital infection in PICU, a good isolation job, reasonable selection of the antibacterial agents according to children's bacterial culture and drug sensitivity, and efficient nursing intervention can prevent from the spreading of the drug-resistance bacteria and control the colonization and spreading of the drug-resistant strains in PICU.

\section{References}

[1] Xu JH (2013). Acinetobacter baumannii susceptibility analysis of the separation of sputum specimens in ICU [J]. China Practical Medical, 11.

[2] Wang Y (2012). The detection rate and resistance rate of Acinetobacter baumannii during the year of 2005 year to 2009 year [J]. Chinese Journal of Nosocomiology, 20.

[3] Xiao YH, Wang J and Zhu Y et al (2010). Nationwide antimicrobial resistance monitoring in the year of 2008 [J]. Chinese Journal of Nosocomiology, 20(16):2377-2383.

[4] Yang LR (2010). Acinetobacter baumannii resistance analysis isolated from the children's test specimens. Chinese Journal of Nosocomiology, 20(22): 3599-3600. 